\title{
Vitamina D y asma: artículo de revisión
}

\author{
ANA MARÍA HERRERA G.*
}

Vitamin D and Asthma, a review

In the last 40 years a progresive decrease of vitamin D levels has been observed in the population due to changes in lifestyles. Low levels of vitamin D have been associated with several diseases, including asthma. Recent studies have shown that low levels of vitamin D in children are correlated with an increase in bronchial reactivity and atopia. In children with an established diagnosis of asthma, low levels of vitamin $D$ is associated with a more severe evolution of asthma, including increased numbers of emergency room visits and hospital admissions, low lung function, increased use of inhaled and systemic corticosteroids and more frequent and severe exacerbations. The role of vitamin D on bronquial asthma is its immunomodulatory action upon the cells of the immune system, inhibiting Th2 type cytokines and IL17 secretion and stimulating IL 10 secretion. In vitro studies suggest a posible role of vitamin D in reverting steroid resistance and airways remodeling.

Key words: Vitamin D, severe asthma, corticosteroids, immune system modulation.

\section{Resumen}

En los últimos 40 años se ha observado una disminución progresiva de los niveles de vitamina $D$ en la población, debido en parte a cambios en los estilos de vida. Bajos niveles vitamina D se han asociado a aumento de varias enfermedades incluyendo el asma bronquial. De acuerdo a la literatura disponible en niños, se ha observado que niveles bajos de vitamina D se correlacionan con aumento de la hiperreactividad bronquial y atopia. En niños con diagnóstico establecido de asma bronquial, bajos niveles de vitamina $D$ se asocian a evolución más severa, incluyendo mayor número de consultas a Servicio de Urgencia y hospitalizaciones, baja función pulmonar, mayor uso de corticoides inhalados y sistémicos y exacerbaciones más frecuentes y severas. La acción de la vitamina $D$ en el asma bronquial estaría dada por su papel inmunomodulatorio sobre las células del sistema inmune, inhibiendo citokinas del tipo Th2, inhibiendo la secreción de IL 17 y estimulando la de IL10. Estudios in vitro sugieren además que la vitamina D tendría cierto papel en revertir la corticoresistencia y la remodelación de la vía aérea.

Palabras clave: Vitamina D, asma severa, corticoesteroides, inmunomodulación.

\section{Introducción}

El organismo adquiere la vitamina D de algunos alimentos como pescado (salmón y atún), queso, mantequilla y yema de huevo. Sin embargo, la fuente más importante de vitamina $\mathrm{D}$ proviene de la producción endógena en la piel a partir del 7 dihidrocolesterol. Este precursor, gracias a la acción de la luz ultravioleta, se convierte en colecalciferol o vitamina $\mathrm{D}^{1}$. A nivel hepático, la enzima 25 hidroxilasa agrega un grupo hidroxilo al colecalciferol transformándolo en 25 hidroxicolecalciferol, que es la forma de depósito de la vitamina $\mathrm{D}$ en el hígado y tejido adiposo. Posteriormente la $25(\mathrm{OH})$ vitamina D llega al riñón donde actúa la 1 alfa hidroxilasa que le agrega un segundo grupo hidroxilo, dando origen finalmente a la forma activa de la vitamina D, la 1, 25 dihidroxicolecalciferol $^{1}$.

La principal función de la vitamina $\mathrm{D}$ y la más conocida es la función esquelética. La vitamina D provee el balance de calcio y fósforo necesario para mantener una adecuada función ósea. Actúa a nivel del intestino delgado aumentando la

* Pediatra broncopulmonar, Clínica Santa María. Santiago. 
absorción de calcio y fósforo y a nivel renal aumentando la reabsorción de calcio en los túbulos proximales. De esta manera se entiende que una deficiencia profunda de vitamina $\mathrm{D}$ dé origen al raquitismo. Esta enfermedad se caracteriza por una alteración en la mineralización de los huesos y del cartílago de crecimiento en los niños, produciendo malformaciones esqueléticas como extremidades inferiores arqueadas, rosario costal y ensanchamiento de las epífisis de los huesos $\operatorname{largos}^{2}$. Se ha demostrado en los últimos años que la enzima que activa a la vitamina $\mathrm{D}$, la 1 alfa hidroxilasa, se encuentra no sólo a nivel renal, sino que también en distintos tejidos, incluyendo el epitelio respiratorio y algunas células del sistema inmune. El receptor de vitamina D (VDR) también está ampliamente distribuido en el organismo, lo que explica las múltiples funciones extraesqueléticas que cumple la vitamina $\mathrm{D}^{1}$. En el último tiempo se han publicado varios trabajos que correlacionan bajos niveles de vitamina $\mathrm{D}$ con distintas enfermedades tales como miopatías, distintos tipos de cáncer, especialmente colorectal, enfermedades autoinmunes como diabetes mellitus y esclerosis múltiple, enfermedades cardiovasculares, neuropsiquiátricas como depresión y Alzheimer y también asma bronquial ${ }^{1,3}$.

\section{Mecanismo de acción de la vitamina $D$}

La vitamina $\mathrm{D}$ es una sustancia liposoluble, por lo que atraviesa fácilmente la membrana celular y posteriormente se transporta al núcleo donde se une a su receptor (VDR). Cuando esto sucede el VDR se asocia a un segundo receptor, el Receptor de Retinoide X, formando un heterodímero que se une a sitios respondedores de vitamina $\mathrm{D}$ (VDRE) directamente en el material genético ${ }^{4}$. Cuando este heterodímero se asocia a moléculas co-activadoras se estimula la síntesis de ciertos genes que darán origen a proteínas y por otro lado al asociarse a moléculas co-represoras ocurre lo contrario. Así la vitamina D actúa primariamente regulando la expresión de genes. Su mecanismo de acción es muy similar al de los corticoides, actuando en el organismo más como una hormona que como una proteína propiamente tal.

\section{Niveles sanguíneos normales de vitamina D}

Los niveles sanguíneos de vitamina $\mathrm{D}$ que se miden son los de 25 hidroxicolecalciferol. Los niveles de vitamina $\mathrm{D}$ pueden ser expresados en nanogramos por mililitro o nanomoles por litro ( $1 \mathrm{nmol} / \mathrm{L}=0,5 \mathrm{ng} / \mathrm{mL}$ ). Se considera que los niveles suficientes de vitamina $\mathrm{D}$ son mayores a $30 \mathrm{ng} / \mathrm{mL}$, insuficientes de 20 a $30 \mathrm{ng} / \mathrm{mL}$ y deficientes $<20 \mathrm{ng} / \mathrm{mL}^{3}$. Debe enfatizarse que estos tres puntos de corte han sido establecidos en base a los niveles requeridos para tener una buena salud esquelética, es decir, para mantener niveles normales de PTH (paratohormona). En la actualidad se desconoce cuáles son los niveles sanguíneos adecuados de vitamina D necesarios para mantener una buena salud desde el punto de vista extra-esquelético.

En los últimos 40 años se ha observado una disminución progresiva de los niveles de vitamina $\mathrm{D}$ en la población. Esto aparentemente sería por cambios en los estilos de vida. Los niños actualmente pasan la mayor parte del tiempo dentro de sus hogares y además la mayoría utiliza en forma rutinaria bloqueador solar por el temor a los efectos adversos de la exposición solar. En Chile hasta el momento no hay estudios publicados acerca de los niveles de vitamina $D$ en nuestra población, pero se podría elucubrar que podrían ser tan bajos como los encontrados en Argentina $(<25 \mathrm{nmol} / \mathrm{L})^{5}$.

\section{Vitamina D y asma}

Desde el punto de vista epidemiológico se ha observado un aumento del número de personas que tienen niveles sanguíneos insuficientes y deficientes de vitamina $\mathrm{D}$ y al mismo tiempo un aumento progresivo de la prevalencia de asma. La pregunta que cabe hacerse es si estos dos fenómenos están relacionados o no. En la literatura hay muchos estudios que asocian niveles de vitamina $\mathrm{D}$ y asma. Con fines didácticos en esta revisión se decidió agruparlos en tres categorías: 1) niveles de vitamina $D$ prenatal y desarrollo posterior de asma; 2) niveles de vitamina $\mathrm{D}$ en la población general y diagnóstico de asma y finalmente; 3 ) niveles de vitamina $\mathrm{D}$ en pacientes con diagnóstico establecido de asma y evolución de la enfermedad.

\section{1) Niveles sanguíneos bajos de vitamina $D$ en el período prenatal y el desarrollo posterior de asma}

Al igual que en la población general, muchas embarazadas tienen niveles bajos de vitamina D. Un estudio belga mostró que $74 \%$ tenían valores insuficientes y $44 \%$ deficientes ${ }^{6}$. La bibliografía disponible en la actualidad es contradictoria al momento de evaluar si los niveles bajos de vitamina $\mathrm{D}$ son un factor de riesgo para el desarrollo futuro de asma en los hijos de estas madres. Por un lado hay varios que demuestran que ingestas normales o altas de vitamina $\mathrm{D}$ durante el emba- 
razo sería un factor protector del desarrollo de asma $^{7-10}$. La limitación de estos estudios es que en ellos no se midió niveles de vitamina $\mathrm{D}$ sino que sólo se hizo un cálculo de la ingesta diaria en base a encuesta alimentaria. Se sabe que los niveles de vitamina D dependen principalmente de la producción endógena y no sólo de la dieta, por lo que es difícil interpretar estos resultados. En otra investigación se midió niveles de vitamina $\mathrm{D}$ a 596 embarazadas a las 32 semanas de gestación y se evalúo la presencia de diagnóstico de asma en sus hijos a los 9 años $^{11}$. En contraposición a los resultados de los estudios anteriores, éste concluye que niveles normales de vitamina $\mathrm{D}$ durante el embarazo (mayores de $75 \mathrm{nmol} / \mathrm{l}$ ) estaría asociado a mayor riesgo de asma a los 9 años (OR: 5,4; IC95\%: 1,09-26,65; $p=0,038$ ). Sin embargo, no se puede sacar conclusiones claras de este estudio ya que el seguimiento a los 9 años fue sólo de un $30 \%$. Finalmente en otra investigación se midió niveles de vitamina $\mathrm{D}$ a 1.585 embarazadas a las 34 semanas de gestación y se evaluó el diagnóstico de asma a los 6 años ${ }^{12}$. A diferencia del estudio anterior en éste el seguimiento fue de $60 \%$. Los autores concluyen que no habría relación alguna entre los niveles prenatales de vitamina D y el diagnóstico de asma a los 6 años.

\section{2) Niveles sanguíneos bajos de vitamina $D$ en la población general como factor de riesgo del desarrollo de asma}

En un estudio transversal realizado en 1.380 niños de 14 años se observó que cerca de $40 \%$ tenía niveles insuficientes de vitamina $\mathrm{D}(<$ a 30 $\mathrm{ng} / \mathrm{ml})^{13}$. Esta población posteriormente se estratificó por nivel de vitamina $\mathrm{D}$ y se observó que no había diferencias en el diagnóstico de asma entre los niños que tenían niveles normales y los que tenían niveles deficientes de vitamina D. Sin embargo, se encontró una correlación positiva entre niveles bajos de vitamina $\mathrm{D}$ y presencia de hiperreactividad bronquial y atopia, especialmente sensibilización al polvo de habitación. En esta cohorte se observó que había algunos aspectos que eran más frecuentes en los pacientes de género masculino como la presencia de hiperreactividad bronquial, atopia y baja función pulmonar. Por lo anterior los autores estratificaron a los niños hombres de acuerdo al nivel de vitamina D, observándose una correlación muy significativa entre niveles bajos de vitamina $\mathrm{D}$ e hiperreactividad bronquial, atopia y sensibilización al polvo de habitación. Se observó una tendencia a tener más frecuentemente diagnóstico de asma en niños varones con niveles bajos de vitamina $\mathrm{D}$, pero esta asociación no alcanzó significancia estadística.

\section{3) Niveles sanguíneos bajos de vitamina $D$ en niños con diagnóstico establecido de asma bronquial y evolución de la enfermedad}

En un estudio transversal se comparó 483 niños asmáticos con 483 niños sanos de la misma edad, observándose que los niveles de vitamina $\mathrm{D}$ eran significativamente más bajos en los niños asmáticos ${ }^{14}$. En otro estudio se evaluó a un grupo de 616 asmáticos de 6 a 14 años y se observó que $28 \%$ de ellos tenían niveles insuficientes de vitamina $\mathrm{D}^{15}$. En los niños que tenían niveles bajos de vitamina $\mathrm{D}$ se demostró que había un aumento significativo de los niveles de IgE total e IgE específica al polvo de habitación. También se evidenció un aumento de la frecuencia de hospitalización y del nivel de hiperreactividad bronquial. Los autores concluyen que los niveles insuficientes de vitamina $\mathrm{D}$ se asocian a un aumento de los marcadores de alergia y a un aumento de la severidad del asma. En otra publicación se estudió a 36 niños con asma severo resistente a tratamiento, 26 niños con asma moderado y 24 controles sanos ${ }^{16}$. En este estudio se observó que los niños con asma severa tenían niveles de vitamina $\mathrm{D}$ significativamente más bajos que los niños con asma moderada y que los controles sanos. Se estableció una correlación positiva entre los niveles de vitamina $\mathrm{D}$ y la función pulmonar medida por $\mathrm{VEF}_{1}$ y CVF. Los niños con asma severa eran los que tenían peor función pulmonar y concomitantemente eran los que tenían niveles más bajos de vitamina $\mathrm{D}$, en comparación con los asmáticos moderados y con los controles sanos. Se observó además una correlación positiva entre niveles de vitamina $\mathrm{D}$ y control del asma medido por cuestionario ACT (Asthma Control Test).

Con respecto a las exacerbaciones de asma se ha observado que éstas son más frecuentes en niños con niveles bajos de vitamina $\mathrm{D}^{16}$. Las crisis serían además más severas, como lo demuestra un trabajo realizado en 1.024 niños de 7 a 10 años con asma persistente leve a moderada. Estos niños fueron seguidos por 4 años en busca de exacerbaciones severas, definidas como aquellas que requieren hospitalización o consulta en Servicio de Urgencia ${ }^{17}$. Durante el período de estudio 352 niños presentaron exacerbaciones severas. Los niños que tenían niveles insuficientes de vitamina D $(35 \%)$ tenían mayor riesgo de hospitalización o consulta a Servicio de Urgencia (OR: 1,5; IC95\%: 1,1-1,9; $\mathrm{p}=0,01)$. Este mayor riesgo se mantuvo incluso luego de ajustarlo por edad, género, índice de masa corporal y diagnóstico de severidad basal. Como los corticoides tienen un efecto protector de las exacerbaciones severas se estratificó el nivel de vitamina D con el uso o 
no uso de este medicamento. Se observó que el mayor riesgo de exacerbación severa lo tenían los niños que estaban sin tratamiento con corticoides inhalados y que al mismo tiempo tenían niveles bajos de vitamina D. Los niños con menor riesgo eran los que estaban recibiendo tratamiento con corticoides inhalados y que además tenían niveles normales de vitamina $\mathrm{D}$. De manera muy interesante se observó que los niños que estaban sin tratamiento con corticoides inhalados, pero que tenían niveles normales de vitamina $\mathrm{D}$ tenían el mismo riesgo de hospitalización y consulta a Servicio de Urgencia que los niños que estaban en tratamiento con corticoides inhalados, pero que tenían niveles bajos de vitamina $\mathrm{D}$. Los resultados de este estudio sugieren que los niveles suficientes de vitamina D conferirían un efecto benéfico adicional al uso de corticoides inhalados, lo que podría tener un efecto terapéutico. Resultados similares al anterior se encontraron en otra investigación realizada en 287 niños asmáticos de 6 a 14 años. Se observó que los que tenían niveles insuficientes de vitamina $\mathrm{D}$ se hospitalizaban y consultaban más frecuentemente al Servicio de Urgencia por exacerbación de asma, en comparación con los que tenían niveles normales. Los autores concluyen que la insuficiencia de vitamina $\mathrm{D}$ se asocia a mayor riesgo de exacerbación severa (OR: 2,6; IC95\%: 1,5-4,9; $\mathrm{p}=0,001)^{18}$.

En la literatura hay disponibles muy pocos trabajos intervencionales con el uso de vitamina D. Uno de ellos es un estudio piloto, randomizado, doble ciego, placebo controlado de 6 meses de duración en 48 niños de 5 a 18 años con diagnóstico reciente de asma ${ }^{19}$. Un grupo de 24 niños recibió Budesonida en polvo seco $800 \mu \mathrm{g}$ al día más placebo y el segundo grupo Budesonida polvo seco en igual dosis más vitamina D $500 \mathrm{UI} /$ día. Se observó que los niños con niveles insuficientes de vitamina $\mathrm{D}$ tenían 8 veces más riesgo de tener exacerbaciones en comparación con los que tenían niveles normales o altos. Durante los 6 meses de tratamiento el número de niños que tuvo exacerbación de asma fue significativamente menor en el grupo que recibió Budesonida más vitamina $\mathrm{D}$ en comparación con el que recibió Budesonida más placebo (17 versus $46 \%$ ). En otro estudio intervencional se evaluó el efecto de la suplementación de vitamina $\mathrm{D}$ en la incidencia de influenza A en escolares en un período de 4 meses $^{20}$. Se estudió a un grupo de 430 niños de 6 a 15 años, 217 recibieron vitamina D $1.200 \mathrm{UI}$ al día y 213 recibieron placebo. Durante los 4 meses de estudio 49 niños fueron diagnosticados con influenza A, $10,8 \%$ en el grupo que recibió vita- mina $\mathrm{D}$ y $18,6 \%$ en el grupo que recibió placebo. Del total de niños 110 tenía diagnóstico de asma, 51 recibió vitamina D y 59 placebo. Se observó que $3,9 \%$ del grupo suplementado con vitamina D tuvo crisis de asma en comparación con $20,3 \%$ en el grupo placebo.

\section{Posibles mecanismos de acción de la Vitamina D en pacientes asmáticos}

La evolución más severa de los niños asmáticos que tienen bajos niveles de vitamina $\mathrm{D}$ estaría dado por el papel inmunomodulatorio de esta vitamina. Como dijimos inicialmente la vitamina $\mathrm{D}$ tiene receptores en varias células del sistema inmune incluyendo linfocitos $\mathrm{B}$, linfocitos $\mathrm{T} y$ células dendríticas ${ }^{1,21}$. Por lo anterior, la vitamina D tendría la capacidad de modular la acción de estas células. Se ha demostrado que los linfocitos $\mathrm{T}$ reguladores son capaces de inhibir la respuesta de tipo Th2 en el asma bronquial ${ }^{22}$ y la vitamina D a su vez es capaz de estimular la acción de estos linfocitos T reguladores y la secreción de IL 10 , una potente citokina antiinflamatoria ${ }^{23,24}$. La vitamina $\mathrm{D}$ puede actuar además a nivel de otra subpoblación de linfocitos, los Th17. La vitamina $\mathrm{D}$ inhibe a los linfocitos Th 17 disminuyendo la secreción de IL $17^{24}$, importante citokina proinflamatoria que se ha asociado a la presencia de asma severo $^{25}$. Así podemos entender que niveles bajos de vitamina D se asocien a niveles altos de IL 17, proinflamatoria y niveles bajos de IL 10 antiinflamatoria. Este desbalance pro inflamatorio que producen los niveles bajos de vitamina $\mathrm{D}$ daría cuenta de la mayor severidad del asma en estos pacientes. En un estudio se midió niveles de IL 6, IL17 e IL 10 a 36 pacientes asmáticos de 6 a 16 años y a 36 controles sanos de la misma edad ${ }^{26}$. En los niños asmáticos se observó un aumento de los niveles de IL 6 y 17 en comparación con los controles sanos y una correlación negativa entre los niveles de IL 17 y vitamina $\mathrm{D}(\mathrm{r}=-0,617$; $\mathrm{p}=0,001)$. El porcentaje de células T reguladoras y la secreción de IL 10 estaban significativamente disminuidas en el grupo de asmáticos, estableciéndose una correlación positiva entre ambas variables y el nivel de vitamina $\mathrm{D}(\mathrm{r}=0,428$ $\mathrm{p}=0,0081)$. De esta manera se objetiva una disminución de la relación IL 10/IL17. Con estos resultados se podría elucubrar que la suplementación con vitamina $\mathrm{D}$ podría revertir la relación IL10/IL17 en los niños asmáticos, teniendo un efecto benéfico en la evolución de la enfermedad.

Con respecto al tratamiento del asma bronquial, está claramente establecido que los corticoides inhalados son el Gold Standard ya que 
hasta la fecha son los únicos capaces de actuar simultáneamente en los distintos niveles de la cascada inflamatoria. El tratamiento administrado debe ser el mínimo posible que permita alcanzar el control de la enfermedad. Al respecto se ha observado que los niños asmáticos con niveles bajos de vitamina D presentan mayor uso de corticoides orales e inhalados en comparación con los que tienen niveles normales ${ }^{16,27}$. Los corticoides ejercen su acción inhibiendo la secreción de citokinas proinflamatorias y aumentando la síntesis de citokinas antiinflamatorias como IL 10. De esta manera podemos ver que los corticoides y la vitamina $\mathrm{D}$ tienen acción sinérgica positiva sobre las células T reguladoras y la secreción de IL 10 . Estudios in vitro han demostrado que la vitamina D tiene efecto ahorrador de corticoides cuando se administra en conjunto con éstos, lo que podría ser de gran valor terapéutico ${ }^{27}$. Los pacientes asmáticos cortico-resistentes tienen incapacidad para secretar cantidades adecuadas de IL 10 en respuesta al uso de corticoides a diferencia de los pacientes cortico-sensibles, que son capaces de aumentar la secreción de esta interleukina en forma dependiente de la dosis ${ }^{28}$. In vitro se ha observado que al administrar simultáneamente vitamina $\mathrm{D}$ y corticoides a linfocitos de pacientes cortico-resistentes, éstos recuperan su capacidad para secretar IL 10 en respuesta a corticoides, en igual proporción que los pacientes corticosensibles ${ }^{29}$. Lo anterior demuestra que la vitamina D eventualmente podría revertir la cortico-resistencia que presentan algunos pacientes asmáticos.

Finalmente se ha visto que en niños con asma severa resistente a tratamiento existe remodelación de la vía aérea, incluyendo engrosamiento de la membrana basal, de la musculatura lisa bronquial y descamación celular. En relación a lo anterior se demostró en un estudio que el grosor de la musculatura lisa bronquial se correlacionaba negativamente con el nivel de vitamina $\mathrm{D}^{16}$. Así bajos niveles de vitamina $\mathrm{D}$ podrían estar influyendo en la remodelación de la vía aérea. Al respecto hay evidencia en la literatura del efecto antiproliferativo celular de la vitamina D. Pruebas in vitro han demostrado que la vitamina $\mathrm{D}$ sería capaz de revertir la hiperplasia de células musculares lisas producidas por ciertos factores de crecimiento, lo que podría estar dando luces acerca del posible rol de la vitamina $\mathrm{D}$ para revertir la remodelación de la vía aérea ${ }^{30}$.

\section{Conclusiones}

Numerosos estudios clínicos demuestran que niños que tienen asma bronquial y niveles bajos de vitamina $\mathrm{D}$ presentan evolución más severa de la enfermedad, incluyendo exacerbaciones frecuentes y graves, mayor número de consultas a Servicio de Urgencia y hospitalizaciones, mayor uso de corticoides tanto inhalados como sistémicos y baja función pulmonar. Sin embargo, la mayoría de estos estudios son de tipo transversal y de asociación por lo que carecen de capacidad para demostrar causalidad. Son escasos los estudios intervencionales que sugieren que la suplementación de vitamina D pudiera disminuir la frecuencia de exacerbaciones de asma. Por otro lado, estudios in vitro han demostrado que la vitamina $\mathrm{D}$ tendría un efecto inmunomodulador, ayudaría a disminuir la cortico-resistencia y a revertir la remodelación de la vía aérea.

Para finalizar podemos decir que toda la información analizada en esta revisión sugiere que tal vez en un futuro la vitamina $D$ pudiera sumarse al arsenal terapéutico habitual del asma bronquial. Sin embargo, antes de poder hacer tal aseveración hace falta una mayor cantidad de estudios intervencionales, multicéntricos, con un número importante de pacientes, que permitan determinar claramente y sin lugar a dudas el papel de la vitamina $\mathrm{D}$ en el asma bronquial.

\section{Bibliografía}

1.- VIDAILHET M, MALLET E, BOCQUET A, BRESSON J L, BRIEND A, CHOURAQUI J P, et al. Vitamin D: Still a topical matter in children and adolescents. A position paper by the Committee on Nutrition of the French Society of Paediatrics. Archives de Pédiatrie 2012; 19: 316-28.

2.- $\quad$ SHAW N J, MUGHAL M Z. Vitamin D and child health Part 1 (skeletal aspects). Arch Dis Child 2013; 0: 1-5.

3.- HOLICK M F. Vitamin D deficiency. N Engl J Med 2007; 357: 266-81.

4.- CALBERG C, CAMPBELL M. Vitamin D receptor signaling mechanisms: Integrated actions of a well-defined transcription factor. Steroids 2013; 78: 127-36.

5.- WAHL D A, COOPER C, EBELING P R, EGGERSDORFER M, HILGER J, HOFFMANN K, et al. A global representation of vitamin D status in healthy populations. Arch Osteoporos 2012; 7: 155-72.

6.- VANDEVIJVERE S, AMSALKHIR S, VAN OYEN H, MORENO-REYES R. High prevalence of vitamin $\mathrm{D}$ deficiency in pregnant women: a national crosssectional survey. Plos One 2012; 7 (8): e43868.

7.- CAMARGO CA J R, RIFAS-SHIMAN S L, LITONJUA A A, RICH-EDWARDS J W, WEISS S T, GOLD D R, et al. Maternal intake of vitamin D during pregnancy and risk of recurrent wheeze in children at $3 \mathrm{y}$ of age. Am J Clin Nutr 2007; 85: 788-95.

8.- DEVEREUX G, LITONJUA A A, TURNER S W, 
CRAIG L C, MCNEILL G, MARTINDALE S, et al. Maternal vitamin D intake during pregnancy and early childhood wheezing. Am J Clin Nutr 2007; 85: 853-9.

9.- ERKKOLA M, KAILA M, NWARU B I, KRONBERGKIPPILA C, AHONEN S, NEVALAINEN J, et al. Maternal vitamin $\mathrm{D}$ intake during pregnancy is inversely associated with asthma and allergic rhinitis in 5-year-old children. Clin Exp Allergy 2009; 39: 875-82.

10.- MIYAKE Y, SASAKI S, TANAKA K, HIROTA Y. Dairy food, calcium and vitamin D intake in pregnancy, and wheeze and eczema in infants. Eur Respir J 2010; 35: 1228-34.

11.- GALE C R, ROBINSON S M, HARVEY N C, JAVAID M K, JIANG B, MARTYN C N, et al. Maternal vitamin $\mathrm{D}$ status during pregnancy and child outcomes. Eur J Clin Nutr 2008; 62: 68-77.

12.- PIKE K C, INSKIP H M, ROBINSON S, LUCAS J $\mathrm{S}$, COOPER C, HARVEY N C, et al. Maternal latepregnancy serum 25-hydroxyvitamin $\mathrm{D}$ in relation to childhood wheeze and atopic outcomes. Thorax 2012; 67: 950-6.

13.- HOLLAMS E M, HART P H, HOLT B J, SERRALHA M, PARSONS F, DE KLERK N H, et al. Vitamin D and atopy and asthma phenotypes in children: a longitudinal cohort study. Eur Respir J 2011; 38: 1320-7.

14.- BENER A, EHLAYEL M S, TULIK M K, HAMID Q. Vitamin D deficiency a strong predictor of asthma in children. Int Arch Allergy Immunol 2012; 157: 168-75.

15.- BREHM J M, CELEDÓN J C, SOTO-QUIROS M E, ÁVILA L, HUNNINGHAKE G M, FORNO E, et al. Serum vitamin D levels and markers of severity of childhood asthma in Costa Rica. Am J Respir Crit Care Med 2009; 179: 765-71.

16.- GUPTA A, SJOUKES A, RICHARDS D, BANYA W, HAWRYLOWICS C, BUSH A. Relationship between serum vitamin D, disease severity, and airway remodeling in children with asthma. Am J Respir Crit Care Med 2011; 184: 1342-9.

17.- BREHM J M, SCHUEMANN B. FUHLBRIGGE A L, HOLLIS B W, STUNK R C, ZEIGER R S, et al. Serum vitamin $\mathrm{D}$ levels and severe asthma exacerbations in the Childhood Asthma Management Program study. J Allergy Clin Immunol 2010; 126: 52-8.

18.- BREHM J M, ACOSTA-PÉREZ E, KLEI L, ROEDER $\mathrm{K}$, BARMADA M, BOUTAOUI N, et al. Vitamin D insufficiency and severe asthma exacerbations in Puerto Rican Children. Am J Respir Crit Care Med 2012; 186 : 140-6.

19.- MAJAK P, OLSZOWIEC-CHLEBNA M, SMEJDA K,
STELMACH I. Vitamin D supplementation in children may prevent asthma exacerbation triggered by acute respiratory infection. J Allergy Clin Immunol 2011; 127: 1294-6.

20.- URASHIMA M, SEGAWA T, OKASAKI M, KURIHARA M, WADA I, IDA H. Randomized trial of vitamin D supplementation to prevent seasonal influenza A in schoolchildren. Am J Clin Nutr 2010; 91: 1255-60.

21.- WANG Y, ZHU J, DE LUCA H F. Where is the vitamin D receptor? Arch Biochem \& Biophys 2012; 523: 123 33.

22.- TAAMS L S, PALMER D B, AKBAR A N, ROBINSON D S, BROWN Z, HOWRYLOWICZ C M. Regulatory $\mathrm{T}$ cells in human disease and their potential for therapeutic manipulation. Immunology 2006; 118: 1-9.

23.- MOORE W, PASCUAL R M. Update in asthma 2009. Am J Respir Crit Care Med 2010; 181: 1181-87.

24.- HEWISON M. Vitamin D and the Immune System: New Perspectives on an Old Theme Endocrinol Metab Clin North Am. 2010; 39: 365-79.

25.- WANG Y H, WILLS-KARP M. The Potential Role of Interleukin-17 in Severe Asthma. Curr Allergy Asthma Rep 2011; 11: 388-94.

26.- MAALMI H, BERRAÏES A, TANGOUR E, AMMAR J, ABID H, HAMZAOUI K, et al. The impact of vitamin $\mathrm{D}$ deficiency on immune $\mathrm{T}$ cells in asthmatic children: a case-control study. Journal of Asthma and Allergy 2012; 5 11-19.

27.- SEARING D, ZHANG I, MURPHY J R, HAUK P J, GOLEVA E, LEUNG D Y. Decreased serum vitamin D levels in children with asthma are associated with increased corticosteroid use. J Allergy Clin Immunol 2010; 125: 995-1000.

28.- HAWRYLOWICZ C, RICHARDS D, LOKE T K, CORRIGAN C, LEE T. A defect in corticosteroid-induced IL-10 production in T lymphocytes from corticosteroidresistant asthmatic patients. J Allergy Clin Immunol 2002; 109: 369-70.

29.- XYSTRAKIS E, KOSUMAKAR S, BOSWELL S, PEEK E, URRY Z, RICHARDS D F, et al. Reversing the defective induction of IL-10-secreting regulatory $\mathrm{T}$ cells in glucocorticoid-resistant asthma patients. J Clin Invest 2006; 116: 146-55.

30.- DAMERA G, FOGLE H W, LIM P, GONCHAROVA E A, ZHAO H, BANERJEE A, et al. Vitamin D inhibits growth of human airway smooth muscle cells through growth factor-induced phosphorylation of retinoblastoma protein and checkpoint kinase 1. British J Pharmacol 2009; 158, 1429-41.

Correspondencia a:

Dra. Ana María Herrera G.

Clínica Santa María

Bellavista 0415. Santiago. Chile

E-mail: aherrera@csm.cl 